\title{
Wirtschaftsstatistik im Wandel - Wichtige gesetzliche Neuerungen in der 19. Legislaturperiode
}

\author{
Christhart Bork $\cdot$ Matthias Pannhorst $\cdot$ Martin Weißenberger
}

Eingegangen: 23. Juli 2021 / Angenommen: 28. Juli 2021 / Online publiziert: 30. August 2021

(C) Der/die Autor(en) 2021

Zusammenfassung Die 19. Legislaturperiode neigt sich dem Ende, was Anlass dazu gibt, einen Rückblick auf die gesetzlichen Neuerungen in dieser Zeit im Bereich der Wirtschaftsstatistik vorzunehmen. Gerade die Corona-Krise hat einmal mehr gezeigt, wie wichtig moderne, zeitnah verfügbare und differenzierte Wirtschaftsstatistiken für die Wirtschaftspolitik sind. Dieser oft (zu) wenig beachtete Politikzweig entwickelt sich seit Jahren äußerst dynamisch. Getrieben von mehreren Großprojekten, die umfassende Reformen bestehender Statistikgesetzgebung anstießen, und stets geprägt von der sich rapide vollziehenden Digitalisierung der amtlichen Statistik waren die gesetzlichen Vorhaben in der Statistikgesetzgebung der 19. Legislaturperiode außergewöhnlich umfangreich und vielschichtig. In diesem Artikel wollen wir die einzelnen Vorhaben in den Blick nehmen und Kontext, Genese sowie besondere Herausforderungen kurz beschreiben.

Schlüsselwörter Außenhandelsstatistik · Bürokratieabbau · Gesetzgebung · Unternehmen · Wirtschaftsstatistik

JEL C80 - E01

\section{Economic statistics in transition - important legal innovations in the 19th legislative period}

Hinweis Die im Beitrag vertretenen Ansichten geben die Meinung der Autoren wieder und entsprechen nicht notwendigerweise den Auffassungen des Bundesministeriums für Wirtschaft und Energie.

Christhart Bork $(\bowtie)$

Referat „Beobachtung, Analysen und Projektionen der gesamtwirtschaftlichen Entwicklung“, Bundesministerium für Wirtschaft und Energie, Berlin, Deutschland

E-Mail: christhart.bork@bmwi.bund.de

Matthias Pannhorst · Martin Weißenberger

Referat „Wachstum, Demografie, Statistik“, Bundesministerium für Wirtschaft und Energie, Berlin, Deutschland 


\section{Die ressortübergreifende Bund-Länder-Arbeitsgruppe zur Reduzierung von Statistikpflichten}

Der Koalitionsvertrag der 19. Legislaturperiode zwischen CDU, CSU und SPD forderte die Einrichtung einer ,Ressortübergreifenden Bund-Länder-Arbeitsgruppe zur Reduzierung von Statistikpflichten“, um weitere Möglichkeiten zur bürokratischen Entlastung von Unternehmen zu identifizieren (CDU et al. 2018). Unter Federführung des Bundesministeriums für Wirtschaft und Energie (BMWi) gehörten dieser Arbeitsgruppe neben den Bundesressorts auch Vertreter der Landeswirtschaftsministerien, die Deutsche Bundesbank und das Statistische Bundesamt an. Die Gruppe hörte die zentralen Hauptverbände der deutschen Wirtschaft an, diese konnten konkrete Vorschläge zur Reduktion von Statistikpflichten einreichen.

Die Arbeitsgruppe stand vor einer großen Herausforderung: Zum einen sind viele Bereiche der Statistik durch europarechtliche Vorgaben geprägt und können nicht ohne weiteres national reduziert werden. Zum anderen entfallen ohnehin lediglich $0,6 \%$ der gesamten bürokratischen Belastungen in Deutschland auf den Bereich Statistik. Trotzdem nehmen Unternehmen Statistikpflichten und andere häufig damit in Verbindung gebrachte Berichtspflichten als wirtschaftliche Einschränkung wahr.

Doch selbst seitens der Wirtschaftsverbände wurde darauf verwiesen, dass Statistiken durchaus nicht aus Selbstzweck heraus erhoben werden. Vielmehr erfüllen sie das Informationsbedürfnis von Politik, Verwaltung und auch der Wirtschaft. So hieß es in einer gemeinsamen Stellungnahme von vier Spitzenverbänden der deutschen Wirtschaft: „Die großen politischen und wirtschaftlichen Herausforderungen (Globalisierung, Digitalisierung, Energie- und Klimawende, zirkuläre Wirtschaft) erfordern nicht ein Weniger, sondern ein Mehr an Informationen, um politisch und unternehmerisch erfolgreich agieren zu können. Daher muss versucht werden, Statistikpflichten effizienter und belastungsärmer als bisher zu gestalten“. Diese Forderung hat sich die Arbeitsgruppe zu eigen gemacht.

Gleichwohl wurde eine umfangreiche Liste von Vorschlägen diskutiert - gegliedert in die drei Hauptbereiche Modernisieren, Digitalisieren und Reduzieren. Die Arbeitsgruppe erarbeitete auf dieser Basis eine Liste von Vorschlägen, wie und wo bürokratische Belastungen abgebaut werden können. Im September 2019 stellte die Ressortübergreifenden Bund-Länder-Arbeitsgruppe zur Reduzierung von Statistikpflichten, BMWi-intern ReBLAuS genannt, nach sechs Sitzungen ihren Abschlussbericht vor. ${ }^{1}$

Die Arbeitsgruppe befasste sich mit der Modernisierung der Registerlandschaft und befürwortete die Einführung einer bundeseinheitlichen Wirtschaftsnummer in Verbindung mit dem Aufbau eines Basisregisters für Unternehmensstammdaten. Dies ist Voraussetzung für die Umsetzung des ,Once-Only“-Prinzips für Unternehmen. Ferner wurde eine Verwaltungsdaten-Informationsplattform vorgeschlagen, mit deren Hilfe Doppelerhebungen künftig identifiziert und vermieden werden können.

Im Bereich der Digitalisierung der Wirtschaftsstatistik schlug die Arbeitsgruppe einige Leuchtturmprojekte vor, mit deren Hilfe die Digitalisierung vorangetrieben

\footnotetext{
1 Der Abschlussbericht ist abrufbar unter https://www.bmwi.de/Redaktion/DE/Downloads/A/ abschlussbericht-reduzierung-von-statistikpflichten.pdf?_blob=publicationFile \&v=6.
} 
werden kann. So kann beispielsweise die Nutzung von Scannerdaten in dem Bereich der Preisstatistik und Einzelhandelsumsatzstatistik zur Verringerung von Erhebungskosten beitragen und die Qualität der Statistiken steigern.

Im Bereich Reduzieren wurden zwölf Statistiken bzw. Teilelemente von Statistiken identifiziert, die künftig entfallen können, ohne dass es zu starken Beeinträchtigungen der Statistiknutzer kommt. Zusammengenommen führen diese zum Teil sehr kleinteiligen Vorschläge zu Entlastungen der Unternehmen in einer Größenordnung von rund 1,5 Mio. $€$ pro Jahr.

\section{Basisregister für Unternehmensstammdaten}

Zentraler Vorschlag der ReBLAuS im Bereich Registermodernisierung war die Schaffung eines Basisregisters für Unternehmensstammdaten in Verbindung mit der Einführung einer bundeseinheitlichen Wirtschaftsnummer. Dieser Vorschlag wurde gemeinsam mit dem Statistischen Bundesamt, der Deutschen Bundesbank, Vertretern betroffener Bundesressorts, einem Vertreter aus den Statistischen Landesämtern und Vertretern der Landeswirtschaftsministerien in einer Unterarbeitsgruppe der ReBLAuS konstruktiv erarbeitet. Zum Ende der Legislaturperiode gelang es dem BMWi Ende April 2021, dem Kabinett einen Gesetzentwurf für ein solches Unternehmensbasisregister (Unternehmensbasisdatenregistergesetz - UBRegG) vorzulegen. Im Juni 2021 konnte das parlamentarische Verfahren abgeschlossen werden und das Gesetz am 15. Juli 2021 in Kraft treten.

Dieses Vorhaben liefert einen wichtigen Beitrag für die Modernisierung, Digitalisierung und Vernetzung der Registerlandschaft in Deutschland. Ziel des Gesamtvorhabens Basisregister ist es, aktuelle und konsistente Stammdaten von Unternehmen zentral vorzuhalten. Unternehmen sollen vor allem von unnötigen Berichtspflichten entlastet werden, indem Mehrfachmeldungen der Stammdaten an unterschiedliche Register künftig vermieden werden, denn die aktuelle Registerlandschaft ist kompliziert und veraltet. Die deutsche Registerlandschaft umfasst rund 120 einzelne Register mit Unternehmensbezug. Geführt werden die Register überwiegend dezentral von unterschiedlichsten Behörden und Registergerichten. Viele Unternehmen sind in mehreren dieser Register erfasst, wobei sich Daten teilweise überschneiden. Ein Austausch von Informationen zwischen den Registern erfolgt üblicherweise nicht. Er ist aufgrund unterschiedlicher bzw. uneinheitlich erfasster und gepflegter Stammdaten zu einzelnen Unternehmen auch nicht ohne weiteres möglich.

Auch eine einheitliche Identifikationsnummer für Unternehmen existiert bislang nicht. Daher ist es zeitaufwändig und fehleranfällig, dasselbe Unternehmen in verschiedenen Registern zu identifizieren, um Daten zu aktualisieren oder im Rahmen der jeweiligen gesetzlichen Vorgaben auszutauschen. Auch die immer wieder erforderliche Pflege und mehrfache Abfrage von Daten führt sowohl auf Seiten der Verwaltung als auch auf Seiten der Unternehmen zu vermeidbarem Aufwand.

Das Basisregister soll alle wirtschaftlich tätigen Einheiten in Deutschland (also natürliche Personen, die wirtschaftlich tätig sind, juristische Personen und Personenvereinigungen) mit ihren Stammdaten (z. B. Namen, Sitz, Geschäftsanschrift, Rechtsform und Wirtschaftszweig) erfassen. Die Unternehmensbasisdaten sollen aus 
angeschlossenen Verwaltungsregistern (Quellregistern, u. a. Handelsregister, Wirtschafts-Identifikationsnummern-Datenbank des Bundeszentralamtes für Steuern) gespeist werden. Sie ersetzen diese Quellregister aber nicht.

Ziel des Gesetzes ist es, die infrastrukturellen Voraussetzungen für den Aufbau eines zentralen Unternehmensbasisregisters zu schaffen. Das Gesetz flankiert dabei vor allem die erste Aufbaustufe eines künftigen zentralen Registers für Unternehmensstammdaten. Es geht zunächst darum, die nötige Infrastruktur zu konzipieren, zu erproben und auszurollen, d.h. den Registeraufbau anzustoßen sowie Schnittstellen zu einzelnen Registern und die Verknüpfung mit der einheitlichen Wirtschaftsnummer herzustellen. Diese erste Phase ist naturgemäß noch nicht mit großen Einsparungen für Unternehmen verbunden. In kommenden Ausbaustufen ist allerdings eine erhebliche Entlastung in Höhe eines dreistelligen Millionenbetrags jährlich für Unternehmen möglich.

Zudem wurden mit dem Gesetz die Voraussetzungen für die Einführung einer bundeseinheitlichen Wirtschaftsnummer geschaffen, um eine register- und verwaltungsübergreifende Identifikation der Unternehmen zu ermöglichen. Als bundeseinheitliche Wirtschaftsnummer dient die Wirtschafts-Identifikationsnummer (W-IdNr.) nach $\S 139 \mathrm{c}$ der Abgabenordnung. Das Bundesministerium für Finanzen wird diese bis spätestens im Jahr 2023 bereitstellen.

Künftig sollen in weiteren Ausbaustufen schrittweise immer mehr Register angebunden werden. Ziel ist es letztendlich, dass das Basisregister die Funktion einer „Datendrehscheibe“ übernehmen kann und die Umsetzung des „Once-Only“Prinzips im Bereich der Unternehmen ermöglicht. Unternehmen können sich dann gegenüber Behörden anhand der bundeseinheitlichen Wirtschaftsnummer eindeutig identifizieren. Behörden können entsprechend ihrer Berechtigungen Daten aus dem Basisregister abrufen und untereinander Daten zu dem Unternehmen austauschen. Dadurch müssen die Daten nicht vom Unternehmen abgefragt werden. Das Basisregister soll Verwaltung schneller, digitaler und moderner machen und sowohl Unternehmen als auch Verwaltung von Bürokratie entlasten.

\section{Verwaltungsdaten-Informationsplattform}

Bislang gab es keinen Überblick darüber, an welcher Stelle welche Daten in welcher Form durch öffentliche Institutionen vorgehalten werden. Doppelungen, Ähnlichkeiten und Inkonsistenzen können daher nicht oder nur sehr schwer erkannt und korrigiert werden. Im Rahmen des EBS-Vorhabens (vgl. unten) wurde im Jahr 2020 die gesetzliche Grundlage für eine von der ReBLAuS vorgeschlagene Verwaltungsdaten-Informationsplattform, die der Normenkontrollrat in die Diskussion einbrachte, geschaffen. Diese im Statistischen Bundesamt geführte Datenbank erfasst ausschließlich Metadaten. Neben allgemeinen Informationen zur Registerführung oder technischen und rechtlichen Aspekten liefert diese Datenbank detaillierte Informationen zu den in den Registern erfassten Merkmalen. Einzelangaben des einzelnen Bürgers oder Unternehmens sind nicht enthalten und damit auch keine personenbezogenen Daten. Im Sommer dieses Jahres soll die VerwaltungsdatenInformationsplattform erstmals öffentlich vorgestellt werden. 
Dadurch wurde ein zentrales Instrument für eine Dateninventur der öffentlichen Hand geschaffen. Die Plattform ist auch ein Instrument zur Reduzierung bestehender Statistikpflichten, denn die Datenbank wird ein Ausgangspunkt bei der Ex-AntePrüfung darüber, ob neue Informationsbedarfe in Statistik und Verwaltung bereits ganz oder teilweise mit anderweitig vorhandenen Daten erfüllt werden können.

Die Plattform kann dafür genutzt werden, bestehende redundante Datenübermittlungen an Verwaltung und Statistik erstmals systematisch herauszuarbeiten. Außerdem trägt die Datenbank zur Reduzierung von Mehrfach-Übermittlungen im Sinne des „Once-Only“-Prinzips bei, da durch sie erst eine Identifikation von Mehrfachübermittlungen möglich wird. Ein solcher Mechanismus entspricht auch dem Prinzip der Datensparsamkeit und der Vermeidung von Redundanzen bei der Führung von Verwaltungsdatenbeständen. Ferner wird die Datentransparenz erhöht und das Open Government unterstützt.

\section{Reform des Preisstatistikgesetzes}

Bereits während der Verhandlungen in der ReBLAuS wurde im Jahr 2019 eine Reform des Preisstatistikgesetzes auf den Weg gebracht, in der Kernvorschläge der Arbeitsgruppe unter dem Stichwort „Digitalisierung der Statistik“ aufgegriffen wurden. Damit wird die Nutzung neuer Erhebungswege ermöglicht, die sich aus der Digitalisierung ergeben und durch die die Qualität der statistischen Ergebnisse gesichert oder, etwa durch die Nutzung von Scannerdaten und die automatisierte Abfrage von Internet-Seiten (,Webscraping“), sogar verbessert werden kann. In das Preisstatistikgesetz wurde eine Regelung aufgenommen, die es ermöglicht Scannerdaten für die Statistik zu nutzen. Hierdurch könnte sich die Revisionsanfälligkeit der bisherigen Erhebung künftig reduzieren. Das BMWi hat gemeinsam mit dem Statistischen Bundesamt, dem Handelsverband Deutschland (HDE) und verschiedenen Unternehmen ein Pilotprojekt initiiert, in dem Scannerdaten erstmals in Deutschland statistisch aufgearbeitet wurden.

Um perspektivisch Scannerdaten auch für die Einzelhandelsumsatzstatistik nutzen zu können, wurde im Rahmen eines späteren Gesetzgebungsverfahrens das Preisstatistikgesetz dahingehend präzisiert, dass diese Daten auch im Rahmen der Konjunkturstatistik für Einzelhandelsumsätze genutzt werden dürfen.

In einem weiteren Gesetzgebungsverfahren wurde eine Regelung eingeführt, durch die das Statistische Bundesamt in den Kreis der Empfänger von Daten der Markttransparenzstelle über die Großhandelspreise für Strom und Gas aufgenommen wird; dadurch können auskunftspflichtige Energieversorgungsunternehmen entlastet und gleichzeitig die Qualität der Preisindizes verbessert werden.

\section{Reform der Verdiensterhebung}

Im Jahr 2019 wurde ferner eine umfassende Überarbeitung des Verdienststatistikgesetzes vorgenommen, die es ermöglicht, die Meldungen zur Verdiensterhebung in weiten Teilen zu digitalisieren und automatisieren. Anstoß zu der Novellierung 
gaben die Mindestlohnkommission und der Bundesverband der Arbeitgeber, die die unten beschriebenen Verbesserungen der Verdiensterhebung einforderten.

Mit der Reform werden die Verdiensterhebung und die Verdienststrukturerhebung zusammengeführt und umgestellt auf eine monatliche verpflichtende Erhebung von Einzeldaten. Die Erhebung sieht eine umfassende Automatisierung und Digitalisierung inklusive dem Einsatz von Künstlicher Intelligenz vor, welche zu einer deutlichen Kostenminimierung beitragen. Im Ergebnis entsteht eine neue, einheitliche und monatlich verfügbare amtliche Datengrundlage, ohne die Unternehmen insgesamt zusätzlich zu belasten. Diese ermöglicht detaillierte Analysen zur Wirkung des Mindestlohns, zur Einkommensverteilung sowie zum sogenannten Gender Pay Gap.

Die Verdiensterhebung hat neben der Politik (z. B. Mindestlohnkommission), den Volkswirtschaftlichen Gesamtrechnungen und der Wissenschaft viele prominente Nutzer: Beispielsweise werden die Diäten der Abgeordneten des Deutschen Bundestags anhand des Nominallohnindex, der aus der Verdiensterhebung gewonnen wird, fortgeschrieben. Unternehmen nutzen die Verdienstindizes im Rahmen von Wertsicherungsklauseln bei mehrjährigen Verträgen.

\section{Einrichtung einer Large Case Unit im Statistischen Bundesamt und Umsetzung des EU-Unternehmensbegriffs}

Gemäß der EU-Einheitenverordnung (VO (EWG) Nr. 696/93) ist in der Statistik die Anwendung des EU-Unternehmensbegriffs vorgesehen. Diese definiert als statistisches Unternehmen die ,kleinste[n] Kombination rechtlicher Einheiten, die eine organisatorische Einheit zur Erzeugung von Waren oder Dienstleistungen bildet und [...] über eine gewisse Entscheidungsfreiheit verfügt" (wirtschaftliche Einheit).

Deutschland hatte den EU-Unternehmensbegriff wegen des hohen Umstellungsaufwands bislang nicht angewandt, die bisher verwendete Unternehmensdefinition stellte auf rechtliche Einheiten ab. Zur Umsetzung ist nun ein sogenanntes Profiling insbesondere bei komplexen Konzernstrukturen erforderlich, so dass einzelne rechtliche Einheiten im Wertschöpfungsprozess zu wirtschaftlichen Einheiten zusammengefasst werden können.

Zum Zweck des Profilings im Bereich von Banken und Versicherungen benötigen das Statistische Bundesamt und die statistischen Ämter der Länder Einzeldaten, die bei den zuständigen Aufsichtsbehörden, namentlich der Deutschen Bundesbank und der Bundesanstalt für Finanzdienstleistungsaufsicht (BaFin), bereits vorliegen, aber nach dem Verwaltungsdatenverwendungsgesetz nicht an die amtliche Statistik weitergegeben werden durften. Eine gesetzliche Anpassung ermöglicht nun die Übermittlung an das Statistische Bundesamt. Durch diese Zweitverwertung bereits vorliegender Daten wurden Doppelerhebungen bei den Unternehmen und damit erhebliche Bürokratiekosten vermieden. Seit 2020 liegen erstmals Strukturergebnisse nach dem neuen Unternehmensbegriff vor, die ein besseres Strukturbild der Wirtschaftsbereiche ermöglichen (Jung und Kaus 2020).

Darüber hinaus stellt die Zunahme der wirtschaftlichen Bedeutung von multinationalen Konzernen im Zuge der Globalisierung die amtliche Statistik vor Herausforderungen. Grenzüberschreitende Umstrukturierungs- und Verlagerungsprozesse 
innerhalb von multinationalen Konzernen können die Qualität der Wirtschaftsstatistiken beeinträchtigen. So kam es z.B. infolge einer Verlagerung von Patentrechten aus Drittstaaten nach Irland zu einem Sprung des irischen Bruttoinlandsprodukts um rund $30 \%$ innerhalb eines Jahres.

Auf europäischer Ebene sollte anhand von Pilotstudien untersucht werden, ob ausgewählte multinationale Unternehmensgruppen in den Volkswirtschaftlichen Gesamtrechnungen der EU-Mitgliedstaaten konsistent und richtig erfasst sind. Im Rahmen der Teilnahme an den EU-Pilotstudien tauschen die statistischen Ämter der jeweils betroffenen Mitgliedstaaten Einzelangaben zu den dort ansässigen Konzernunternehmen aus, wofür in Deutschland (im Gegensatz zu allen anderen Mitgliedstaaten) jedoch eine Rechtsgrundlage fehlte. Eine weitere EU-Pilotstudie dient der Überprüfung des Bruttonationaleinkommens (BNE), das Bemessungsgrundlage zur Eigenmittelausstattung der EU ist. An der Richtigkeit der Berechnung des BNE hat Deutschland als größter Nettobeitragszahler in den EU-Haushalt ein erhebliches Interesse.

Es wurde eine befristete Regelung des grenzüberschreitenden Einzeldatenaustauschs geschaffen, so dass sich Deutschland angemessen an diesen EU-Pilotstudien beteiligen konnte. Diese beiden Vorhaben wurden in dem „Gesetz zur ergänzenden Regelung der statistischen Verwendung von Verwaltungsdaten und zur Regelung der Übermittlung von Einzelangaben zu multinationalen Unternehmensgruppen an statistische Stellen“ im Jahr 2018 umgesetzt.

Die Pilotstudie zur Analyse von Konzernstrukturen war erfolgreich, so dass eine permanente Einheit geschaffen wurde, die statistische Zuordnungsfragen von sehr großen Berichtseinheiten klärt. Dies erfolgte im Zusammenhang mit der Modernisierung des Außenhandelsstatistikgesetzes, in dem ein Gesetz zur dauerhaften Sicherstellung der Qualität von multinationalen Unternehmensgruppen in den Volkswirtschaftlichen Gesamtrechnungen und den Wirtschaftsstatistiken aufgenommen wurde. Im Statistischen Bundesamt wurde eine sogenannte LCU (Large Cases Unit) etabliert.

\section{Umsetzung der Regulation on European Business Statistics (EBS)}

Mit der Rahmenverordnung EBS (European Business Statistics, früherer Name FRIBS: Framework Regulation for Integrated Business Statistics) wurde auf EUEbene im Jahr 2019 eine unionsweite Harmonisierung der Unternehmensstatistik und eine breitere Einbeziehung wirtschaftlicher Aktivitäten auf den Weg gebracht. Dies bietet die Chance auf eine bessere Qualität sowie intraeuropäische Vergleichbarkeit von statistischen Daten. Die damit einhergehenden Änderungen beinhalten sowohl Erweiterungen der Lieferverpflichtungen als auch methodische Anpassungen bei der Erhebung und der Verarbeitung der zu liefernden Angaben. Insbesondere werden Regelungen für ein vollständigeres Abbild des Dienstleistungssektors in der amtlichen Statistik getroffen, welcher rund 70\% der Bruttowertschöpfung in Deutschland ausmacht. In der Konjunkturstatistik wird als Darstellungseinheit ein Umstieg auf die fachliche Einheit (,Kind-of-Activity-Unit“) für alle Wirtschaftsbereiche vollzogen, bisher erfolgte für die Bereiche Handel und Dienstleistungen eine 
Darstellung auf Unternehmensebene. Die Änderung ermöglicht aussagekräftigere Konjunkturergebnisse, da umsatzstarke Unternehmen mit verschiedenen Tätigkeiten nun nicht mehr nur einem Wirtschaftszweig zugeordnet werden, sondern ihr Umsatz entsprechend auf die verschiedenen Wirtschaftszweige aufgeteilt werden kann. Zudem wurde der Erfassungsbereich um einige Wirtschaftsbereiche, u. a. Grundstücksund Wohnungswesen, erweitert. Mit einem Artikelgesetz, das die EBS-Rahmenverordnung in deutsches Recht umsetzt, wurde auch ein monatlicher Produktionsindex im Dienstleistungsbereich eingeführt, um ein zeitnahes Abbild des Dienstleistungssektors in den Konjunkturstatistiken zu erhalten. Bei den strukturellen Unternehmensstatistiken wurde die Lieferverpflichtung um die Wirtschaftsbereiche „Erziehung und Unterricht“, „Gesundheits- und Sozialwesen“, „Kunst, Unterhaltung und Erholung“, „Erbringung von sonstigen überwiegend persönlichen Dienstleistungen“ und um Teile des Wirtschaftsbereichs „Erbringung von Finanz- und Versicherungsdienstleistungen“" erweitert, so dass letzterer nun vollständig abgedeckt ist. Damit bildet die strukturelle Unternehmensstatistik künftig nahezu alle Wirtschaftsbereiche ab. Darüber hinaus wurde die bereits erwähnte, seit Jahren vorgeschriebene EUUnternehmensdefinition in den deutschen Unternehmensstatistiken verankert.

\section{Reform des Außenhandelsstatistikgesetzes}

Im Zuge der nationalen Verankerung von EBS wurde der Bereich des Außenhandels aufgrund der hohen Komplexität und Kleinteiligkeit der entsprechenden Regelungen sowie abweichender zeitlicher Umsetzungserfordernisse nicht im vorgenannten Umsetzungsgesetz geregelt; dies erfolgte im Jahr 2021 in einem separaten Gesetzgebungsverfahren.

Der inhaltliche Schwerpunkt bei der Umsetzung von EBS im Außenhandel liegt auf der Einführung des sogenannten Einstromverfahrens: Nach den neuen europäischen Vorgaben sind die Mitgliedstaaten künftig nicht mehr verpflichtet, Daten über Wareneingänge aus anderen Mitgliedstaaten an das Statistische Amt der Europäischen Union (Eurostat) zu melden; stattdessen wird mit der EBS-Verordnung eine Verpflichtung zum Austausch von Einzeldaten des innergemeinschaftlichen Warenverkehrs, des sogenannten Intrahandels, zwischen den Statistikämtern der Mitgliedstaaten eingeführt. Dies führt perspektivisch zu deutlichen Entlastungen der Unternehmen von Berichtspflichten. Bis eine hinreichende Datenqualität unter dem neuen Verfahren sichergestellt ist, sieht die deutsche Umsetzung gegenwärtig eine komplementäre Einführung zur bestehenden Vorgehensweise vor. Erste Daten unter dem neuen Verfahren sollen im Jahr 2022 vorliegen.

Weiterhin erfolgte anlässlich der im Zuge der EBS-Umsetzung anstehenden Überarbeitungen eine grundsätzliche Neukonzeptionierung des Außenhandelsstatistikgesetzes; die bislang geltenden Regelungen der Außenhandelsstatistik stammten aus der Zeit vor der Einführung des europäischen Binnenmarktes.

Die Änderungen an der zum Außenhandelsstatistikgesetz gehörenden Durchführungsverordnung (Außenhandelsstatistik-Durchführungsverordnung) umfassten fachstatistische Anpassungen an die aktuellen Gegebenheiten und Konkretisierungen einzelner Bestimmungen des neuen Außenhandelsstatistikgesetz. 


\section{Fazit}

Insgesamt hat die Arbeit der ReBLAuS die Statistikgesetzgebung der Legislatur maßgeblich geprägt: Das BMWi strengte mehrere Gesetzgebungsvorhaben an, die Vorschläge der Arbeitsgruppe umsetzten. Letztlich konnten damit in dieser Legislaturperiode alle Vorschläge der Arbeitsgruppe gesetzgeberisch verankert werden. In allen Verfahren wurde stets Wert daraufgelegt, der deutschen Wirtschaft keine zusätzlichen Belastungen aufzuerlegen.

Die Ergebnisse der Arbeitsgruppe verdeutlichen aber auch, dass eine Reduzierung der bürokratischen Belastungen im Bereich der Wirtschaftsstatistik nur noch begrenzt möglich ist, zumal über die bislang oft praktizierten Wege, etwa die Anpassung von Meldeschwellen oder des Stichprobenumfangs. Während in der Legislatur das Volumen der Entlastungen durch die Reduzierung von Statistikpflichten absolut betrachtet nicht sehr hoch ist, können aber durch die Einführung der bundeseinheitlichen Wirtschaftsnummer in Verbindung mit dem Aufbau des Basisregisters für Unternehmensstammdaten Einsparungen von Bürokratielasten in der Größenordnung eines jährlich dreistelligen Millionenbetrags erreicht werden.

So dürfte auch in der Zukunft eine weitere Entlastung im Bereich der Statistik vor allem durch das aktive Voranbringen neuer Projekte, maßgeblich in den Feldern Modernisierung und Digitalisierung, zu erreichen sein. Dies ist kein per se neues Thema (vgl. etwa Pannhorst 2017), gewinnt aber zunehmend an Dringlichkeit und politischem Momentum.

Funding Open Access funding enabled and organized by Projekt DEAL.

Open Access Dieser Artikel wird unter der Creative Commons Namensnennung 4.0 International Lizenz veröffentlicht, welche die Nutzung, Vervielfältigung, Bearbeitung, Verbreitung und Wiedergabe in jeglichem Medium und Format erlaubt, sofern Sie den/die ursprünglichen Autor(en) und die Quelle ordnungsgemäß nennen, einen Link zur Creative Commons Lizenz beifügen und angeben, ob Änderungen vorgenommen wurden.

Die in diesem Artikel enthaltenen Bilder und sonstiges Drittmaterial unterliegen ebenfalls der genannten Creative Commons Lizenz, sofern sich aus der Abbildungslegende nichts anderes ergibt. Sofern das betreffende Material nicht unter der genannten Creative Commons Lizenz steht und die betreffende Handlung nicht nach gesetzlichen Vorschriften erlaubt ist, ist für die oben aufgeführten Weiterverwendungen des Materials die Einwilligung des jeweiligen Rechteinhabers einzuholen.

Weitere Details zur Lizenz entnehmen Sie bitte der Lizenzinformation auf http://creativecommons.org/ licenses/by/4.0/deed.de.

\section{Literatur}

CDU, CSU, SPD (2018) Ein neuer Aufbruch für Europa. Eine neue Dynamik für Deutschland. Ein neuer Zusammenhalt für unser Land. Koalitionsvertrag zwischen CDU, CSU und SPD. https://www. bundesregierung.de/resource/blob/656734/847984/5b8bc23590d4cb2892b31c987ad672b7/201803-14-koalitionsvertrag-data.pdf. Zugegriffen: 22. Juli 2021 (19. Legislaturperiode, Berlin)

Jung S, Kaus W (2020) Unternehmensstrukturstatistiken und Statistik für Kleine und Mittlere Unternehmen nach dem EU-Unternehmensbegriff. Ergebnisse für 2018. Wirtsch Stat 2020(6):68-81

Pannhorst M (2017) Modernisierung der Wirtschaftsstatistik. Potenziale der Digitalisierung nutzen und Rolle der Politikevaluation stärken. Schlaglichter Wirtschaftspolitik 2017(12):24-29 
Hinweis des Verlags Der Verlag bleibt in Hinblick auf geografische Zuordnungen und Gebietsbezeichnungen in veröffentlichten Karten und Institutsadressen neutral. 